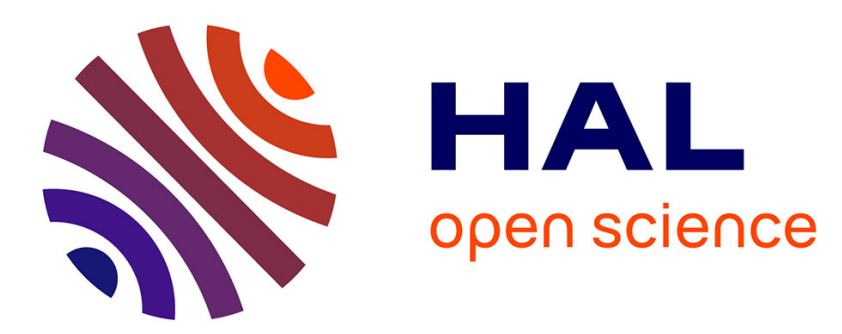

\title{
Generalized Multiplicative Extended Kalman Filter for Aided Attitude and Heading Reference System
}

\author{
Philippe Martin, Erwan Salaün
}

\section{To cite this version:}

Philippe Martin, Erwan Salaün. Generalized Multiplicative Extended Kalman Filter for Aided Attitude and Heading Reference System. 2010 AIAA Guidance, Navigation, and Control Conference, Aug 2010, Toronto, Canada. pp.AIAA 2010-8300. hal-00555580

HAL Id: hal-00555580

https://hal-mines-paristech.archives-ouvertes.fr/hal-00555580

Submitted on 13 Jan 2011

HAL is a multi-disciplinary open access archive for the deposit and dissemination of scientific research documents, whether they are published or not. The documents may come from teaching and research institutions in France or abroad, or from public or private research centers.
L'archive ouverte pluridisciplinaire HAL, est destinée au dépôt et à la diffusion de documents scientifiques de niveau recherche, publiés ou non, émanant des établissements d'enseignement et de recherche français ou étrangers, des laboratoires publics ou privés. 


\title{
Generalized Multiplicative Extended Kalman Filter for Aided Attitude and Heading Reference System
}

\author{
Philippe Martin * \\ Mines ParisTech, 75272 Paris Cedex 06, France \\ Erwan Salaün ${ }^{\dagger}$ \\ Georgia Institute of Technology, Atlanta, GA 30332-0150, USA
}

\begin{abstract}
In this paper, we propose a "Generalized Multiplicative Extended Kalman Filter" (GMEKF) to estimate the position and velocity vectors and the orientation of a flying rigid body, using measurements from lowcost Earth-fixed position and velocity, inertial and magnetic sensors. Thanks to well-chosen state and output errors, the gains and covariance equations converge to constant values on a much bigger set of trajectories than equilibrium points as it is the case for the standard Multiplicative Extended Kalman Filter (MEKF). We recover thus the fundamental properties of the Kalman filter in the linear case, especially the convergence and optimality properties, for a large set of trajectories, and it should result in a better convergence of the estimation. We illustrate the good performance and the nice properties of the GMEKF on simulation and on experimental comparisons with a commercial system.
\end{abstract}

\section{Nomenclature}

$\omega \quad$ Instantaneous angular velocity vector, $\mathrm{rad} / \mathrm{s}$

$\omega_{b} \quad$ Constant vector bias on gyroscopes, $\mathrm{rad} / \mathrm{s}$

$\omega_{m} \quad$ Instantaneous angular velocity vector measured by the gyroscopes, $\mathrm{rad} / \mathrm{s}$

$A \quad$ Constant gravity vector in North-East-Down coordinates, $A=g e_{3}, \mathrm{~m} / \mathrm{s}^{2}$

$a \quad$ Specific acceleration vector (all the non-gravitational forces divided by the body mass), $\mathrm{m} / \mathrm{s}^{2}$

$a_{m} \quad$ Specific acceleration vector measured by the accelerometers, $\mathrm{m} / \mathrm{s}^{2}$

$a_{s} \quad$ Positif constant scaling factor on accelerometers

$B \quad$ Earth magnetic field in NED coordinates, $\mathrm{T}$

$e_{i} \quad$ Unit vectors pointing respectively North, East, Down, $\mathrm{i}=1,2,3$

$h_{b} \quad$ Constant scalar bias on altitude measurement provided by the barometric sensor, $\mathrm{m}$

$q \quad$ Unit quaternion representing the orientation of the body-fixed frame with respect to the Earth-fixed frame

$V, X \quad$ Velocity and position vectors of the center of mass with respect to the Earth-fixed frame, $\mathrm{m} / \mathrm{s}, \mathrm{m}$

\section{Introduction}

Aircraft, especially Unmanned Aerial Vehicles (UAV), commonly need to know their orientation, velocity and position to be operated, whether manually or with computer assistance. When cost or weight is an issue, using very accurate inertial sensors for "true" (i.e. based on the Schuler effect due to a non-flat rotating Earth) inertial navigation is excluded. Instead, low-cost systems -sometimes called aided Attitude and Heading Reference Systems (aided AHRS) - rely on light and cheap "strapdown" gyroscopes, accelerometers and magnetometers "aided" by position and velocity sensors (provided for example in body-fixed coordinates by an air-data or Doppler radar system, or in Earth-fixed coordinates by a GPS engine). The various measurements are then "merged" according to the motion equations of the aircraft assuming a flat non-rotating Earth, with some filtering algorithm. Although many filtering techniques have been proposed and studied to design observers for aided AHRS (e.g. invariant nonlinear observers, ${ }^{1-3}$

*Professor, Centre Automatique et Systèmes, philippe.martin@mines-paristech. fr, AIAA Member

${ }^{\dagger}$ Postdoctoral Fellow, School of Aerospace Engineering, erwan. salaun@gatech. edu, AIAA Member. 
Sigma-point Kalman filtering, ${ }^{4,5}$ adaptative filtering ${ }^{6,7}$ ), the Extended Kalman Filter (EKF) is still the most widely used approach to design such estimators. To design an EKF, the system is seen as a stochastic differential equation,

$$
\begin{aligned}
& \dot{x}=f(x, u)+M w \\
& y=h(x, u)+N v,
\end{aligned}
$$

where $x, u, y$ belong to an open subset of $\mathbb{R}^{n} \times \mathbb{R}^{m} \times \mathbb{R}^{p} ; w, v$ are independent white gaussian noises of size $n$ and $p$, and $M, N$ are square matrices. The input $u$ and output $y$ are known signals, and the state $x$ must be estimated. An estimation $\hat{x}(t)$ of $x(t)$ is then computed by the EKF

$$
\begin{aligned}
& \dot{\hat{x}}=f(\hat{x}, u)+K \cdot(y-h(\hat{x}, u)) \\
& \dot{P}=A P+P A^{T}+M M^{T}-P C^{T}\left(N N^{T}\right)^{-1} C P,
\end{aligned}
$$

with $K=P C^{T}\left(N N^{T}\right)^{-1}, A=\partial_{1} f(\hat{x}, u)$ and $C=\partial_{1} h(\hat{x}, u)\left(\partial_{i}\right.$ means the partial derivative with respect to the $i^{t h}$ argument). The rationale is to compute the gain $K$ as in a linear Kalman filter since the estimation error $\Delta x=\hat{x}-x$ satisfies up to higher order terms the linear equation

$$
\Delta \dot{x}=(A-K C) \Delta x-M w+K N v .
$$

Of course the convergence of the EKF is not guaranteed in general as in the linear case, see e.g. Ref. 8 for some (local) convergence results.

Another drawback of this "linear" approach is that it is often not adapted to the specific context of aerospace engineering. Indeed in this field, it is customary to use a unit quaternion $q$ to represent the attitude of the aircraft, instead of Euler angles, since it provides a global parametrization of the body orientation, and is well-suited for calculations and computer simulations. In this case, the "linear" approach of the EKF does not respect the geometry of the quaternion space: the standard linear correction term does not preserve the norm of the quaternion, whereas the standard linear error $\hat{q}-q$ does not really make sense for quaternions. Therefore ad hoc modifications of the EKF are used, in particular the so-called Multiplicative EKF (MEKF), introduced in Refs. 9-11, see also Refs. 12,13. The idea is to respect the geometry of the quaternion space, by using for the quaternion estimation a multiplicative correction term which preserves the unit norm, and by computing the error equation with the error $q^{-1} * \hat{q}$ (or equivalently $\left.\hat{q}^{-1} * q=\left(q^{-1} * \hat{q}\right)^{-1}\right)$. However, the MEKF leads to matrices $A$ and $C$ that depend on the trajectory. We recover then the fundamental properties of the Kalman filter in the linear case (especially the convergence properties) only at the equilibrium points of the system: convergence issues may happen in many situations, even if the UAV follows a "smooth" trajectory.

In this paper we propose a modification of the MEKF, so-called "Generalized Multiplicative EKF" (GMEF), to overcome the limitations (regarding the convergence properties) of the MEKF. The GMEKF comes directly from the method developed in Ref. 14 to construct an Invariant Extended Kalman Filter (IEKF) for nonlinear systems possessing symmetries (built on the ideas developed in Refs. 15-17). The main benefit of the IEKF is that the matrices $A$ and $C$ are constant on a much bigger set of trajectories (so-called "permanent trajectories"16) than equilibrium points as it is the case for the EKF (and for the MEKF). Near such trajectories, we are back to the "true", i.e. linear, Kalman filter where convergence is guaranteed. Informally, this means the IEKF should in general converge at least around any slowly-varying permanent trajectory, rather than just around any slowly-varying equilibrium point for the EKF. Moreover, the filter proposed in this paper supersedes the examples developed in Ref. 14 (i.e. Right and Left IEKF).

The paper is organized as follows. In Section II, we first describe the considered system. Then, we design in Section III a "standard" MEKF and we compare its main features with the GMEKF designed in Section IV. Finally, we illustrate the good performance and the nice properties of the GMEKF on simulation (in Section V) and on experimental comparisons with a commercial system (in Section VI).

\section{Physical equations and measurements}

\section{A. Motion equations}

The motion of a flying rigid body (assuming the Earth is flat and defines an inertial frame) is described by

$$
\begin{aligned}
\dot{q} & =\frac{1}{2} q * \omega \\
\dot{V} & =A+q * a * q^{-1} \\
\dot{X} & =V .
\end{aligned}
$$


The first equation describes the kinematics of the body, the second is Newton's force law. For more details about this section and about the quaternions, see any good textbook on aircraft modeling, for instance Ref. 18, and appendix for useful formulas used in this paper.

\section{B. Measurements}

We use four triaxial sensors, yielding twelve scalar measurements: 3 gyros measure $\omega_{m}=\omega+\omega_{b}$, where $\omega_{b}$ is a constant vector bias; 3 accelerometers measure $a_{m}=a_{s} a$, where $a_{s}>0$ is a constant scaling factor; 3 magnetometers measure $y_{B}=q^{-1} * B * q$; the position and velocity vectors $X$ and $V$ are provided by the navigation solutions $y_{X}$ and $y_{V}$ of a GPS engine (the GPS velocity is obtained from the carrier phase and/or Doppler shift data, and not by differentiating the GPS position, hence is of rather good quality). A barometric sensor provides also a measure of the altitude $y_{h}=\left\langle X, e_{3}\right\rangle-h_{b}$, where $h_{b}$ is a constant scalar bias. There is some freedom in the modeling of the sensors imperfections, see Ref. 3 for a discussion. All these measurements are of course also corrupted by noise.

It is reasonable to assume each scalar sensor is corrupted by an additive gaussian white noise with identical variance for each of the three scalar sensors constituting a triaxial sensor, and all the noises mutually independent (this is technologically motivated for the accelerometers, gyros and magnetic sensors, though much more questionable for the GPS engine). Hence we can see each triaxial sensor as corrupted by a "coordinate-free vector noise" whose coordinates are gaussian in the body frame as well as the Earth frame (or any other smooth time-varying frame). Indeed, the mean and the auto-correlation time of such a noise is not affected by a (smoothly) time-varying rotation.

\section{The considered system}

To design our observers we therefore consider the system

$$
\begin{aligned}
\dot{q} & =\frac{1}{2} q *\left(\omega_{m}-\omega_{b}\right) \\
\dot{V} & =A+\frac{1}{a_{s}} q * a_{m} * q^{-1} \\
\dot{X} & =V \\
\dot{\omega}_{b} & =0 \\
\dot{a}_{s} & =0 \\
\dot{h}_{b} & =0,
\end{aligned}
$$

where $\omega_{m}$ and $a_{m}$ are seen as known inputs, together with the output

$$
\left(\begin{array}{l}
y_{V} \\
y_{X} \\
y_{h} \\
y_{B}
\end{array}\right)=\left(\begin{array}{c}
V \\
X \\
\left\langle X, e_{3}\right\rangle-h_{b} \\
q^{-1} * B * q
\end{array}\right) .
$$

This system is observable provided $B \times\left(q * a_{m} * q^{-1}\right) \neq 0$ since all the state variables can be recovered from the known quantities $\omega_{m}, a_{m}, y_{V}, y_{X}, y_{h}, y_{B}$ and their derivatives: see Ref. 3 for details.

\section{Standard Multiplicative Extended Kalman Filter}

We start with the design of a Multiplicative EKF in the spirit of Refs. 9-11, see also Refs. 12, 13. 


\section{A. Problem setting}

We consider the noise enters the system as

$$
\begin{aligned}
\dot{q} & =\frac{1}{2} q *\left(\omega_{m}-\omega_{b}\right)+q * M_{q} w_{q} \\
\dot{V} & =A+\frac{1}{a_{s}} q * a_{m} * q^{-1}+q * M_{V} w_{V} * q^{-1} \\
\dot{X} & =V+M_{X} w_{X} \\
\dot{\omega}_{b} & =M_{\omega} w_{\omega} \\
\dot{a}_{s} & =M_{a} w_{a} \\
\dot{h}_{b} & =M_{h} w_{h},
\end{aligned}
$$

and the output as

$$
\left(\begin{array}{c}
y_{V} \\
y_{X} \\
y_{h} \\
y_{B}
\end{array}\right)=\left(\begin{array}{c}
V+N_{V} v_{V} \\
X+N_{X} v_{X} \\
\left\langle X, e_{3}\right\rangle-h_{b}+N_{h} v_{h} \\
q^{-1} * B * q+N_{B} v_{B}
\end{array}\right),
$$

with $M_{q}, M_{V}, M_{X}, M_{\omega}, N_{V}, N_{X}, N_{B}$ diagonal matrices, and $M_{a}, M_{h}, N_{h}$ scalars. The driving and observation noises are thus consistent with a scalar additive noise on each individual sensor.

\section{B. MEKF equations}

The MEKF then takes the form

$$
\begin{aligned}
\dot{\hat{q}} & =\frac{1}{2} \hat{q} *\left(\omega_{m}-\hat{\omega}_{b}\right)+\hat{q} * K_{q} E \\
\dot{\hat{V}} & =A+\frac{1}{\hat{a}_{s}} \hat{q} * a_{m} * \hat{q}^{-1}+K_{V} E \\
\dot{\hat{X}} & =\hat{V}+K_{X} E \\
\dot{\hat{\omega}}_{b} & =K_{\omega} E \\
\dot{\hat{a}}_{s} & =K_{a} E \\
\dot{\hat{h}}_{b} & =K_{h} E,
\end{aligned}
$$

where the output error is given by

$$
E=\left(\begin{array}{c}
\hat{y}_{V}-y_{V} \\
\hat{y}_{X}-y_{X} \\
\hat{y}_{h}-y_{h} \\
\hat{y}_{B}-y_{B}
\end{array}\right)=\left(\begin{array}{c}
\hat{V}-V-N_{V} v_{V} \\
\hat{X}-X-N_{X} v_{X} \\
\left\langle\hat{X}-X, e_{3}\right\rangle-\hat{h}_{b}+h_{b}-N_{h} v_{h} \\
\hat{q}^{-1} * B * \hat{q}-q^{-1} * B * q-N_{B} v_{B}
\end{array}\right) .
$$

But for Eq. (20), the MEKF has the form of a standard EKF.

We consider the state error

$$
\left(\begin{array}{c}
\mu \\
v \\
\chi \\
\beta \\
\alpha \\
\lambda
\end{array}\right)=\left(\begin{array}{c}
q^{-1} * \hat{q} \\
\hat{V}-V \\
\hat{X}-X \\
\hat{\omega}_{b}-\omega_{b} \\
\hat{a}_{s}-a_{s} \\
\hat{h}_{b}-h_{b}
\end{array}\right) .
$$


A tedious but simple computation yields the error system

$$
\begin{aligned}
\dot{\mu} & =-\frac{1}{2} \beta * \mu+\mu \times \hat{I}_{\omega}-M_{q} w_{q} * \mu+\mu * K_{q} E \\
\dot{v} & =\hat{I}_{a}-\frac{1}{\hat{a}_{s}-\alpha} \hat{q} \mu^{-1} * a_{m} * \mu * \hat{q}^{-1}-\mu * \hat{q} * M_{V} w_{V} * \hat{q}^{-1} * \mu^{-1}+K_{V} E \\
\dot{\chi} & =v-M_{X} w_{X}+K_{X} E \\
\dot{\beta} & =-M_{\omega} w_{\omega}+K_{\omega} E \\
\dot{\alpha} & =-\alpha M_{a} w_{a}+\alpha K_{a} E \\
\dot{\lambda} & =-M_{h} w_{h}+K_{h} E
\end{aligned}
$$

where the output error is rewritten as

$$
E=\left(\begin{array}{c}
v-N_{V} v_{V} \\
\chi-N_{X} v_{X} \\
\left\langle\chi, e_{3}\right\rangle-\lambda-N_{h} v_{h} \\
\hat{I}_{B}-\mu * \hat{I}_{B} * \mu^{-1}-N_{B} v_{B}
\end{array}\right),
$$

and $\hat{I}_{\omega}=\omega_{m}-\hat{\omega}_{b}, \hat{I}_{a}=\frac{1}{\hat{a}_{s}} \hat{q} * a_{m} * \hat{q}^{-1}$ and $\hat{I}_{B}=\hat{q}^{-1} * B * \hat{q}$.

We next linearize this error system around $(\bar{\mu}, \bar{v}, \bar{\chi}, \bar{\beta}, \bar{\alpha}, \bar{\lambda})=(1,0,0,0,0,0)$, i.e. the estimated state equals the actual state, drop all the quadratic terms in noise and infinitesimal state error (see Ref. 14 for mathematical justification), and eventually find

$$
\left(\begin{array}{l}
\delta \dot{\mu} \\
\delta \dot{v} \\
\delta \dot{\chi} \\
\delta \dot{\beta} \\
\delta \dot{\alpha} \\
\delta \dot{\lambda}
\end{array}\right)=(A-K C)\left(\begin{array}{l}
\delta \mu \\
\delta v \\
\delta \chi \\
\delta \beta \\
\delta \alpha \\
\delta \lambda
\end{array}\right)-M\left(\begin{array}{l}
w_{q} \\
w_{V} \\
w_{X} \\
w_{\omega} \\
w_{a} \\
w_{h}
\end{array}\right)+K N\left(\begin{array}{c}
v_{V} \\
v_{X} \\
v_{h} \\
v_{B}
\end{array}\right)
$$

This linearized error system has the desired form (5) with

$$
\begin{aligned}
A & =\left(\begin{array}{cccccc}
-\hat{I}_{\omega \times} & 0_{33} & 0_{33} & -\frac{1}{2} I_{3} & 0_{31} & 0_{31} \\
-2 \hat{I}_{a \times} R(\hat{q}) & 0_{33} & 0_{33} & 0_{33} & -\hat{I}_{a} & 0_{31} \\
0_{33} & I_{3} & 0_{33} & 0_{33} & 0_{31} & 0_{31} \\
0_{33} & 0_{33} & 0_{33} & 0_{33} & 0_{31} & 0_{31} \\
0_{13} & 0_{13} & 0_{13} & 0_{13} & 0_{11} & 0_{11} \\
0_{13} & 0_{13} & 0_{13} & 0_{13} & 0_{11} & 0_{11}
\end{array}\right) \\
C & =\left(\begin{array}{cccccc}
0_{33} & I_{3} & 0_{33} & 0_{33} & 0_{31} & 0_{31} \\
0_{33} & 0_{33} & I_{3} & 0_{33} & 0_{31} & 0_{31} \\
0_{13} & 0_{13} & e_{3}^{T} & 0_{13} & 0_{11} & -I_{1} \\
2 \hat{I}_{B \times} & 0_{33} & 0_{33} & 0_{33} & 0_{31} & 0_{31}
\end{array}\right) \\
M & =\operatorname{Diag}\left(M_{q}, R(\hat{q}) M_{V}, M_{X}, M_{\omega}, M_{a}, M_{h}\right) \\
N & =\operatorname{Diag}\left(N_{V}, N_{X}, N_{h}, N_{B}\right) \\
K & =-\left(K_{q}, K_{V}, K_{X}, K_{\omega}, K_{a}, K_{h}\right)^{T},
\end{aligned}
$$

where the matrices $I_{\times}$and $R(q)$ are defined by $I_{\times} u:=I \times u$ and $R(q) u:=q * u * q^{-1}$ for all for $u \in \mathbb{R}^{3}$. Similarly to the standard EKF, the matrix gain $K$ is now computed as $K=P C^{T}\left(N N^{T}\right)^{-1}$ with $P$ defined by (4).

\section{Features of the MEKF}

SOUND GEOMETRIC STRUCTURE FOR THE QUATERNION ESTIMATION EQUATION by construction, Eq. (20) preserves the unit norm of the estimated quaternion, since $K_{q} E$ is a vector of $\mathbb{R}^{3}$ (see Appendix). 
POSSIBLE CONVERGENCE ISSUES IN MANY SITUATIONS indeed, the matrices $A$ and $C$ used for computing the gain matrix $K$ are constant only in level flight, i.e. $\hat{I}_{\omega} \simeq 0$ and $\hat{I}_{a} \simeq-A$, because of the trajectory-dependent terms $R(\hat{q})$ in $A$ and $C$.

\section{Generalized Multiplicative Extended Kalman Filter}

Following the method proposed in Ref. 14, we now design a Generalized MEKF.

\section{A. Problem setting}

We consider the noise enters the system as

$$
\begin{aligned}
\dot{q} & =\frac{1}{2} q *\left(\omega_{m}-\omega_{b}\right)+M_{q} w_{q} * q \\
\dot{V} & =A+\frac{1}{a_{s}} q * a_{m} * q^{-1}+M_{V} w_{V} \\
\dot{X} & =V+M_{X} w_{X} \\
\dot{\omega}_{b} & =q^{-1} * M_{\omega} w_{\omega} * q \\
\dot{a}_{s} & =a_{s} M_{a} w_{a} \\
\dot{h}_{b} & =M_{h} w_{h},
\end{aligned}
$$

and the output as

$$
\left(\begin{array}{c}
y_{V} \\
y_{X} \\
y_{h} \\
y_{B}
\end{array}\right)=\left(\begin{array}{c}
V+N_{V} v_{V} \\
X+N_{X} v_{X} \\
\left\langle X, e_{3}\right\rangle-h_{b}+N_{h} v_{h} \\
q^{-1} *\left(B+N_{B} v_{B}\right) * q
\end{array}\right)
$$

with $M_{q}, M_{V}, M_{X}, M_{\omega}, N_{V}, N_{X}, N_{B}$ diagonal matrices, and $M_{a}, M_{h}, N_{h}$ scalars. Notice the noise on $y_{B}$ is different from the noise in (19): it is additive in body axes rather than in Earth axes; the same frame change appears for driving noise in Eqs. 26,27,29, see section II for a tentative physical justification.

\section{B. GMEKF equations}

The GMEKF then takes the form

$$
\begin{aligned}
\dot{\hat{q}} & =\frac{1}{2} \hat{q} *\left(\omega_{m}-\hat{\omega}_{b}\right)+K_{q} E * \hat{q} \\
\dot{\hat{V}} & =A+\frac{1}{\hat{a}_{s}} \hat{q} * a_{m} * \hat{q}^{-1}+K_{V} E \\
\dot{\hat{X}} & =\hat{V}+K_{X} E \\
\dot{\hat{\omega}}_{b} & =\hat{q}^{-1} * K_{\omega} E * \hat{q} \\
\dot{\hat{a}}_{s} & =\hat{a}_{s} K_{a} E \\
\dot{\hat{h}}_{b} & =K_{h} E,
\end{aligned}
$$

where the output error is given by

$$
E=\left(\begin{array}{c}
\hat{y}_{V}-y_{V} \\
\hat{y}_{X}-y_{X} \\
\hat{y}_{h}-y_{h} \\
B-\hat{q} * y_{B} * \hat{q}^{-1}
\end{array}\right)=\left(\begin{array}{c}
\hat{V}-V-N_{V} v_{V} \\
\hat{X}-X-N_{X} v_{X} \\
\left\langle\hat{X}-X, e_{3}\right\rangle-\hat{h}_{b}+h_{b}-N_{h} v_{h} \\
B-\hat{q} * q^{-1} *\left(B+N_{B} v_{B}\right) * q * \hat{q}^{-1}
\end{array}\right) .
$$


We consider the state error

$$
\left(\begin{array}{c}
\mu \\
v \\
\chi \\
\beta \\
\alpha \\
\lambda
\end{array}\right)=\left(\begin{array}{c}
\hat{q} * q^{-1} \\
\hat{V}-V \\
\hat{X}-X \\
q *\left(\hat{\omega}_{b}-\omega_{b}\right) * q^{-1} \\
\frac{\hat{a}_{s}}{a_{s}} \\
\hat{h}_{b}-h_{b}
\end{array}\right)
$$

The error system is

$$
\begin{aligned}
& \dot{\mu}=-\frac{1}{2} \mu * \beta-\mu * M_{q} w_{q}+K_{q} E \mu \\
& \dot{v}=\hat{I}_{a}-\alpha \mu^{-1} * \hat{I}_{a} * \mu-M_{V} w_{V}+K_{V} E \\
& \dot{\chi}=v-M_{X} w_{X}+K_{X} E \\
& \dot{\beta}=\left(\mu^{-1} * \hat{I}_{\omega} * \mu\right) \times \beta+M_{q} w_{q} \times \beta-M_{\omega} w_{\omega}+\mu^{-1} * K_{\omega} E * \mu \\
& \dot{\alpha}=-\alpha M_{a} w_{a}+\alpha K_{a} E \\
& \dot{\lambda}=-M_{h} w_{h}+K_{h} E,
\end{aligned}
$$

where the output error is rewritten as

$$
E=\left(\begin{array}{c}
v-N_{V} v_{V} \\
\chi-N_{X} v_{X} \\
\left\langle\chi, e_{3}\right\rangle-\lambda-N_{h} v_{h} \\
B-\mu *\left(B+N_{B} v_{B}\right) * \mu^{-1}
\end{array}\right)
$$

and $\hat{I}_{\omega}=\hat{q} *\left(\omega_{m}-\hat{\omega}_{b}\right) * \hat{q}^{-1}, \hat{I}_{a}=\frac{1}{\hat{a}_{s}} \hat{q} * a_{m} * \hat{q}^{-1}$.

We next linearize this error system around $(\bar{\mu}, \bar{v}, \bar{\chi}, \bar{\beta}, \bar{\alpha}, \bar{\lambda})=(1,0,0,0,0,0)$, drop all the quadratic terms in noise and infinitesimal state error, and eventually find

$$
\left(\begin{array}{c}
\delta \dot{\mu} \\
\delta \dot{v} \\
\delta \dot{\chi} \\
\delta \dot{\beta} \\
\delta \dot{\alpha} \\
\delta \dot{\lambda}
\end{array}\right)=(A-K C)\left(\begin{array}{l}
\delta \mu \\
\delta v \\
\delta \chi \\
\delta \beta \\
\delta \alpha \\
\delta \lambda
\end{array}\right)-M\left(\begin{array}{l}
w_{q} \\
w_{V} \\
w_{X} \\
w_{\omega} \\
w_{a} \\
w_{h}
\end{array}\right)+K N\left(\begin{array}{l}
v_{V} \\
v_{X} \\
v_{h} \\
v_{B}
\end{array}\right)
$$

which has the desired form (5) with

$$
\begin{aligned}
A & =\left(\begin{array}{cccccc}
0_{33} & 0_{33} & 0_{33} & -\frac{1}{2} I_{3} & 0_{31} & 0_{31} \\
-2 \hat{I}_{a \times} & 0_{33} & 0_{33} & 0_{33} & -\hat{I}_{a} & 0_{31} \\
0_{33} & I_{3} & 0_{33} & 0_{33} & 0_{31} & 0_{31} \\
0_{33} & 0_{33} & 0_{33} & \hat{I}_{\omega \times} & 0_{31} & 0_{31} \\
0_{13} & 0_{13} & 0_{13} & 0_{13} & 0_{11} & 0_{11} \\
0_{13} & 0_{13} & 0_{13} & 0_{13} & 0_{11} & 0_{11}
\end{array}\right) \\
C & =\left(\begin{array}{cccccc}
0_{33} & I_{3} & 0_{33} & 0_{33} & 0_{31} & 0_{31} \\
0_{33} & 0_{33} & I_{3} & 0_{33} & 0_{31} & 0_{31} \\
0_{13} & 0_{13} & e_{3}^{T} & 0_{13} & 0_{11} & -I_{1} \\
2 B_{\times} & 0_{33} & 0_{33} & 0_{33} & 0_{31} & 0_{31}
\end{array}\right) \\
M & =\operatorname{Diag}\left(M_{q}, M_{V}, M_{X}, M_{\omega}, M_{a}, M_{h}\right) \\
N & =\operatorname{Diag}\left(N_{V}, N_{X}, N_{h}, N_{B}\right) \\
K & =-\left(K_{q}, K_{V}, K_{X}, K_{\omega}, K_{a}, K_{h}\right)^{T} .
\end{aligned}
$$

As usual the matrix gain $K$ is computed as $K=P C^{T}\left(N N^{T}\right)^{-1}$ with $P$ defined by (4). 


\section{Features of the GMEKF}

SOUND GEOMETRIC STRUCTURE FOR THE QUATERNION ESTIMATION EQUATION by construction, Eq. (33) preserves the unit norm of the estimated quaternion, since $K_{q} E$ is a vector of $\mathbb{R}^{3}$ (see Appendix).

LARGER EXPECTED DOMAIN OF CONVERGENCE the matrices $A$ and $C$ used for computing the gain matrix $K$ are constant not only in level flight but also on every trajectory defined by constant $\hat{I}_{\omega}, \hat{I}_{a}$.

SYMMETRY-PRESERVING STRUCTURE since the GMEKF is a specific Invariant EKF, ${ }^{14}$ rotations, translations and scaling in the appropriated frames leave the error system unchanged, unlike the MEKF: it is meaningful from an engineering point of view, and it should result to a physically more "sensible" convergence of the estimations.

\section{Simulation results}

We first illustrate the behavior of the proposed filters on simulations. The noises $w_{i}, v_{i}$ are independent normally distributed random 3-dimensional vectors with mean 0 and variance 1. The tuning of the MEKF and GMEKF is made via the choice of covariance matrices $M_{q}=0.1 I_{3}, M_{V}=0.01 I_{3}, M_{X}=0.1 I_{3}, M_{\omega}=0.001 I_{3}, M_{a}=0.01, M_{h}=0.01$, $N_{V}=0.1 I_{3}, N_{X}=0.1 I_{3}, N_{h}=0.1, N_{B}=0.1 I_{3}$. The (scaled) Earth magnetic field is taken as $B=\left(\begin{array}{lll}1 & 0 & 1\end{array}\right)^{T}$ (roughly the value in France).

To enforce $\|\hat{q}\|=1$ despite numerical round off, we systematically add the term $k\left(1-\|\hat{q}\|^{2}\right) \hat{q}$ in the estimated quaternion equation (otherwise the norm would slowly drift), which is a standard trick in numerical integration with quaternions. For instance for the GMEKF, we take

$$
\dot{\hat{q}}=\frac{1}{2} \hat{q} *\left(\omega_{m}-\hat{\omega}_{b}\right)+K_{q} E * \hat{q}+k\left(1-\|\hat{q}\|^{2}\right) \hat{q} .
$$

We have used $k=1$ (this value is not critical).

The system follows a trajectory $\mathscr{T}_{1}$, which is quite representative of a small UAV flight. The MEKF and GMEKF are initialized with the same values. Both filters give correct estimations after the initial transient, see Fig. 1.

We now show the GMEKF gain matrix $K$ becomes as expected constant on the trajectory $\mathscr{T}_{1}$, while the MEKF gain does not, see Fig. 2. This is remarkable since $\mathscr{T}_{1}$ is far from being an equilibrium point.

Finally we illustrate the invariance property of the GMEKF: both EKF are initialized with three different initial conditions having the same initial state error. The MEKF behavior does depend on the initial conditions, while the GMEKF behavior does not, see Fig. 3 (only the norm of the velocity error $e_{v}=\|v\|$ is displayed, but similar results are obtained for other state errors).

\section{Experimental results}

We now compare the behavior of our observer with the commercial INS-GPS device MIDG II from Microbotics Inc. For each experiment we first save the raw measurements from the MIDG II gyros, accelerometers and magnetic sensors (at a $50 \mathrm{~Hz}$ refresh rate), the position and velocity provided by the navigation solutions of its GPS engine (at a 5 $\mathrm{Hz}$ refresh rate) and the raw measurements from a barometer module Intersema MS5534B (at a $12.5 \mathrm{~Hz}$ refresh rate). A microcontroller on a development kit communicate with these devices and send the measurements to a computer via the serial port (see Fig. 4). On Matlab Simulink we feed offline the observer with these data and then compare the estimations of the observer to the estimations given by the MIDG II (computed according to the user manual by some Kalman filter). In order to have similar behaviors and considering the units of the raw measurements provided by the MIDG II, we have chosen $M_{q}=1 e-3 I_{3}, M_{V}=5 e-3 I_{3}, M_{X}=0.01 I_{3}, M_{\omega}=1 e-7 I_{3}, M_{a}=2 e-6, M_{h}=2 e-4$, $N_{V}=5 e-3 I_{3}, N_{X}=0.1 I_{3}, N_{h}=0.1, N_{B}=1 e-3 I_{3}$, and $k=1$.

We wait a few minutes until the biases reach constant values, then move the system in all directions. The GMEKF and the MIDG II give similar results (see Fig. 5). On Fig. 5(d) it is in fact $h_{b}+168 m$ that is represented to plot $a_{s}$ and $h_{b}$ on the same axis. We can also notice on Fig. 5(b) that the estimation of $V_{Z}$ given by our observer seems to be closer to the real value than the estimation provided by the MIDG II: we know that we let the system motionless at $t=42 s$, which is coherent with our estimated $V_{Z}$. In Fig. 6, we also plot the evolution of some coefficients of the gain matrix $K(t)$ (the same as in Section $\mathrm{V}$ ). As expected, the gain matrix remains (almost) constant during the experiment, even if the trajectory followed by the system is far from the equilibrium point. 


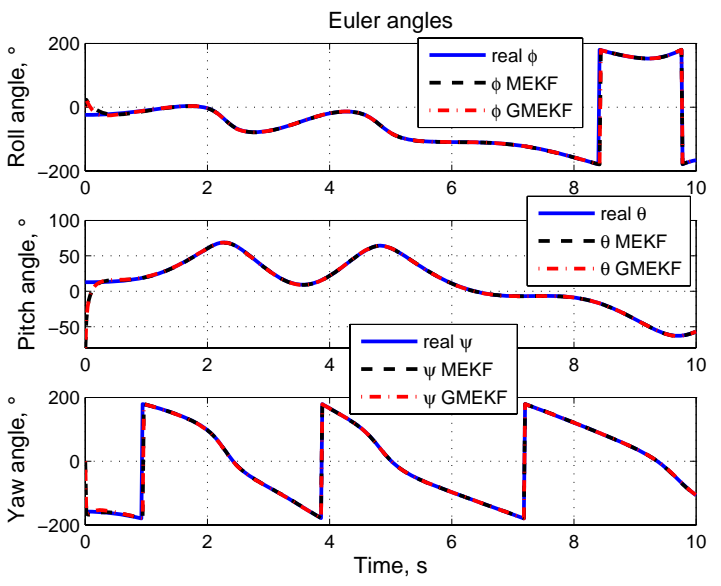

(a) Estimated Euler angles
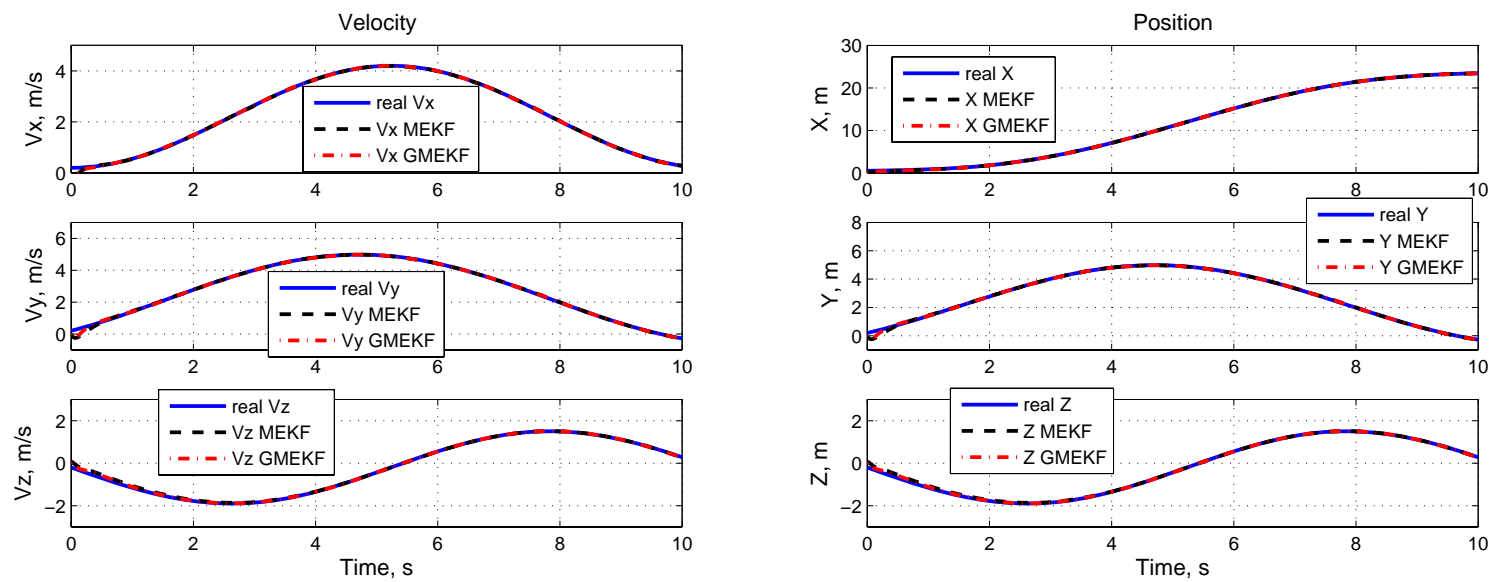

(b) Estimated velocity

(c) Estimated position

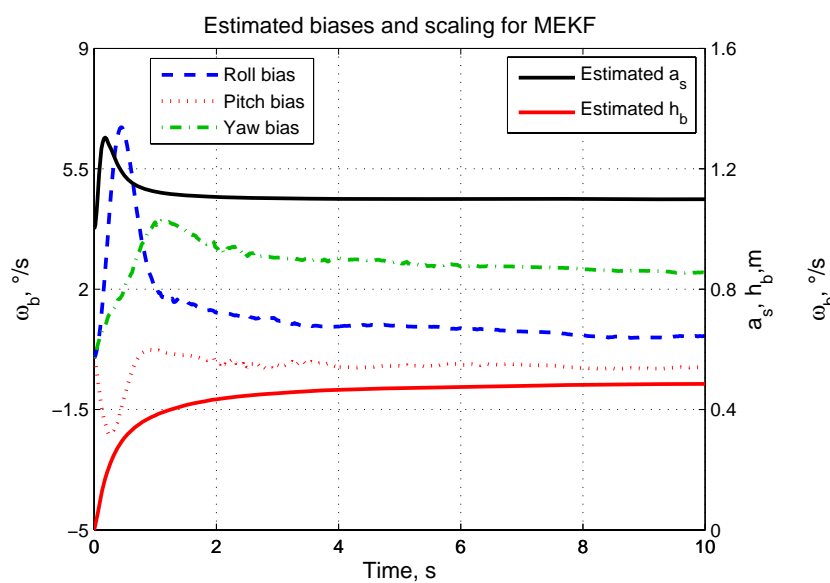

(d) Estimated biases and scaling (MEKF)

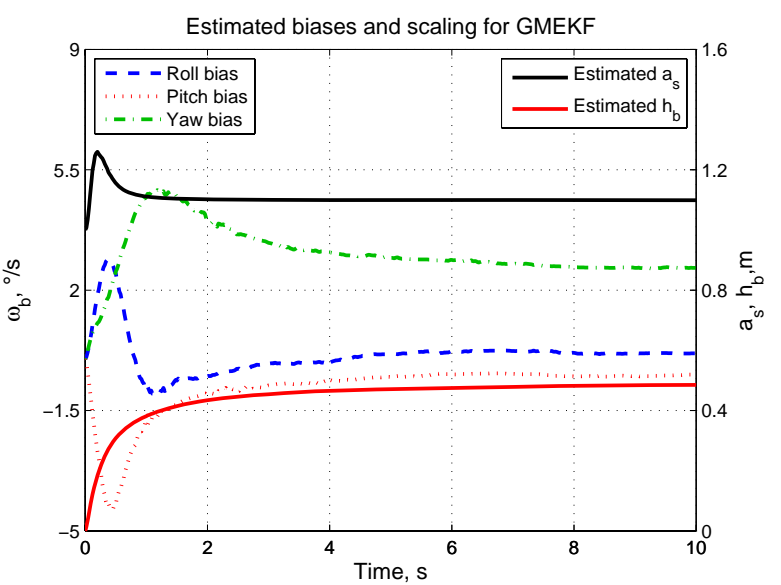

(e) Estimated biases and scaling (GMEKF)

Figure 1. Simulation: Comparison MEKF / GMEKF 

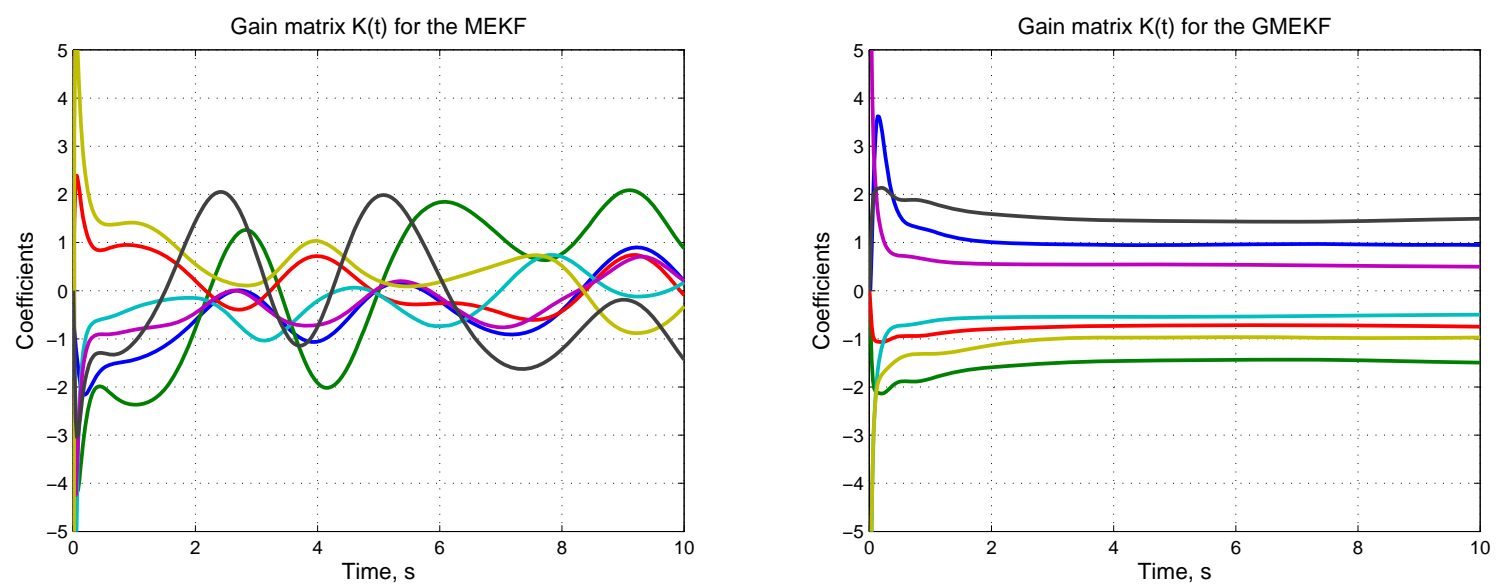

Figure 2. Simulation: Evolution of gain matrix $K$ (MEKF left, GMEKF right)
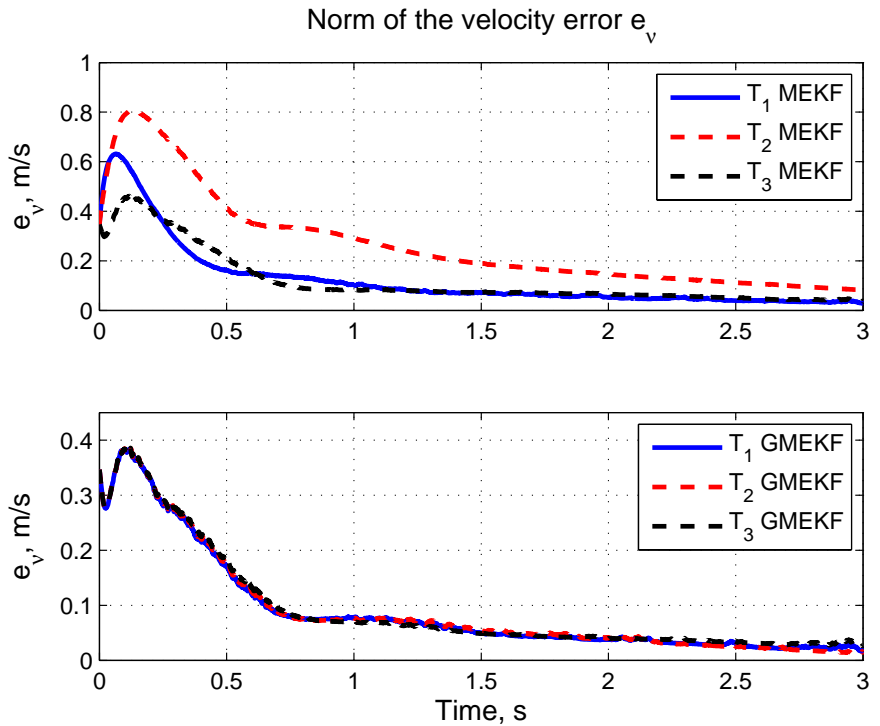

Figure 3. Simulation: Evolution of $e_{v}=\|v\|$ for three initial conditions 


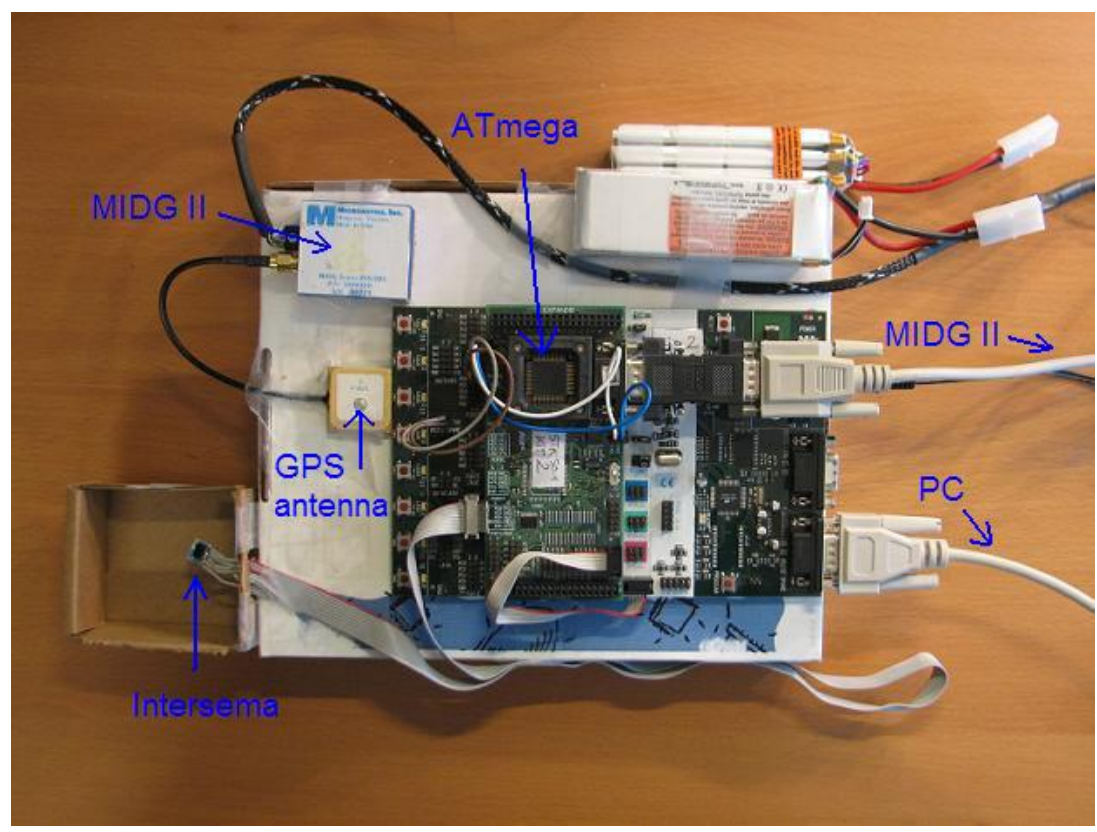

Figure 4. Experimental protocol

\section{Appendix: Quaternions}

Thanks to their four coordinates, quaternions provide a global parametrization of the orientation of a rigid body (whereas a parametrization with three Euler angles necessarily has singularities). Indeed, to any quaternion $q$ with unit norm is associated a rotation matrix $R_{q} \in S O(3)$ by

$$
q^{-1} * \vec{p} * q=R_{q} \cdot \vec{p} \quad \text { for all } \vec{p} \in \mathbb{R}^{3} .
$$

A quaternion $p$ can be thought of as a scalar $p_{0} \in \mathbb{R}$ together with a vector $\vec{p} \in \mathbb{R}^{3}$,

$$
p=\left(\begin{array}{c}
p_{0} \\
\vec{p}
\end{array}\right) .
$$

The (non commutative) quaternion product $*$ then reads

$$
p * q \triangleq\left(\begin{array}{c}
p_{0} q_{0}-\vec{p} \cdot \vec{q} \\
p_{0} \vec{q}+q_{0} \vec{p}+\vec{p} \times \vec{q}
\end{array}\right) .
$$

The unit element is $e \triangleq\left(\begin{array}{l}1 \\ \overrightarrow{0}\end{array}\right)$, and $(p * q)^{-1}=q^{-1} * p^{-1}$.

Any scalar $p_{0} \in \mathbb{R}$ can be seen as the quaternion $\left(\begin{array}{c}p_{0} \\ \overrightarrow{0}\end{array}\right)$, and any vector $\vec{p} \in \mathbb{R}^{3}$ can be seen as the quaternion $\left(\begin{array}{l}0 \\ \vec{p}\end{array}\right)$. We systematically use these identifications in the paper, which greatly simplifies the notations.

We have the useful formulas

$$
\begin{gathered}
p \times q \triangleq \vec{p} \times \vec{q}=\frac{1}{2}(p * q-q * p) \\
(\vec{p} \cdot \vec{q}) \vec{r}=-\frac{1}{2}(p * q+q * p) * r .
\end{gathered}
$$

If $q$ depends on time, then $\dot{q}^{-1}=-q^{-1} * \dot{q} * q^{-1}$. 

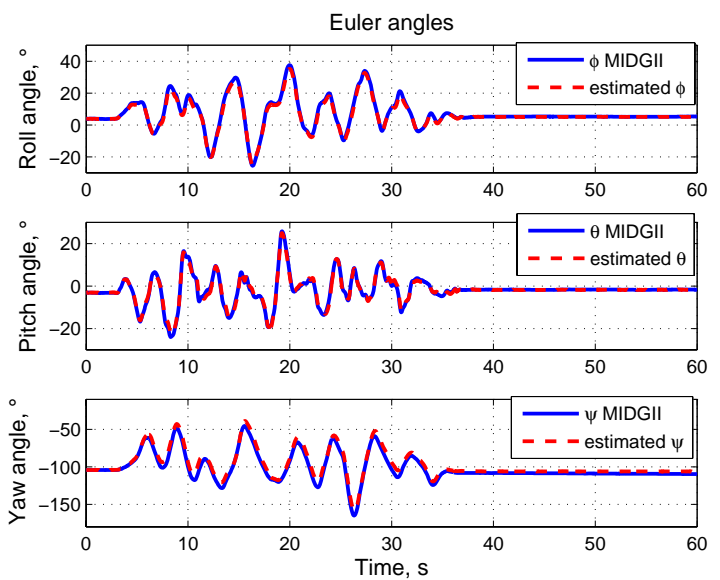

(a) Estimated Euler angles
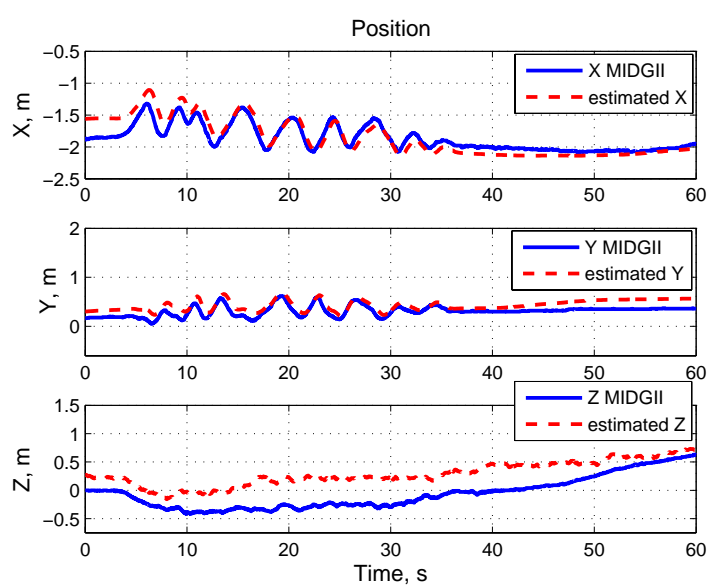

(c) Estimated position
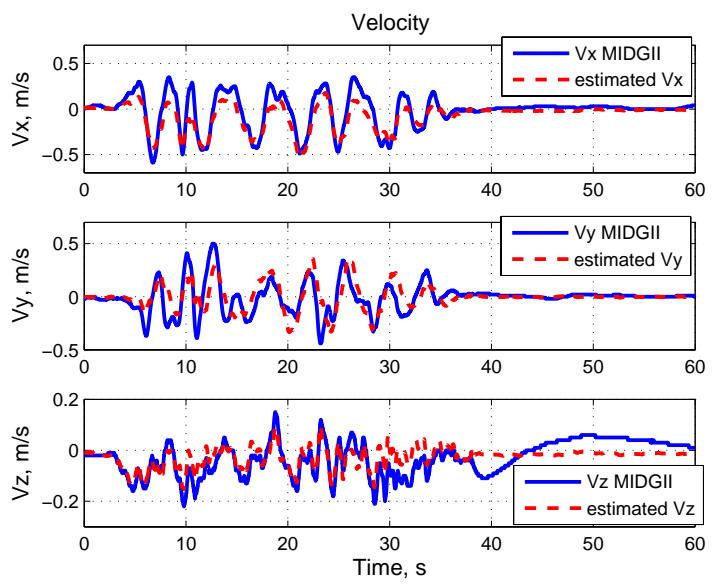

(b) Estimated velocity

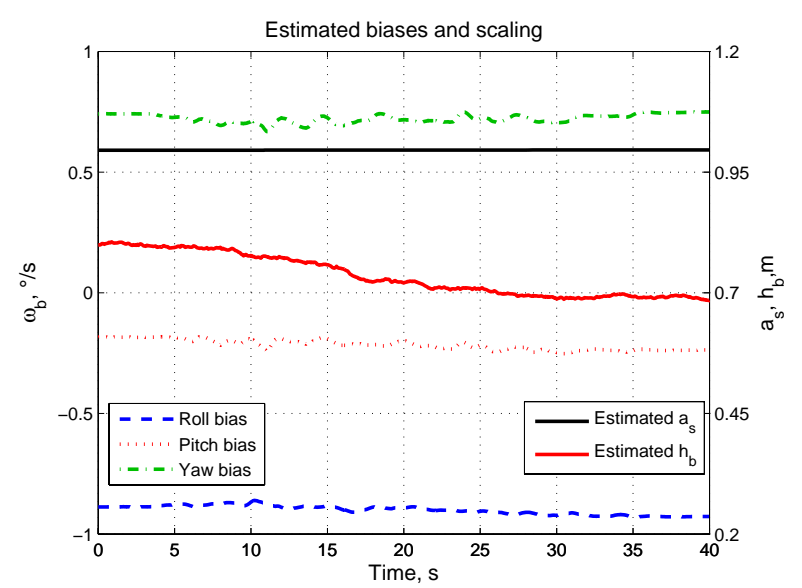

(d) Estimated biases and scaling

Figure 5. Experiment : Dynamic behavior

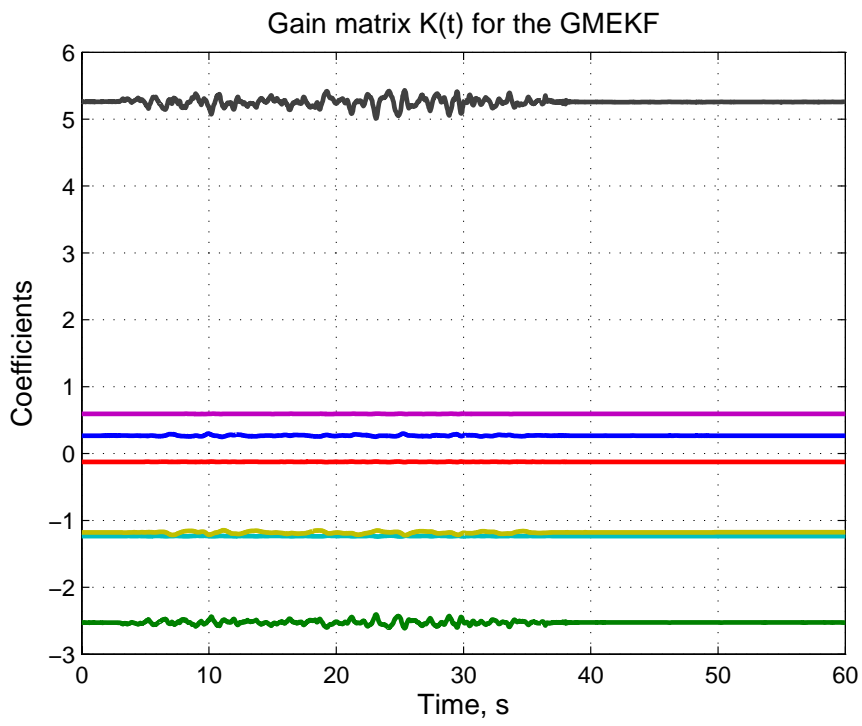

Figure 6. Experiment: Evolution of gain matrix $K$ 
Finally, consider the differential equation $\dot{q}=q * u+v * q$ where $u, v$ are vectors $\in \mathbb{R}^{3}$. Let $q^{T}$ be defined by $\left(\begin{array}{c}q_{0} \\ -\vec{q}\end{array}\right)$. Then $q * q^{T}=\|q\|^{2}$. Therefore,

$$
\overbrace{q * q^{T}}^{i}=q *\left(u+u^{T}\right) * q^{T}+\|q\|^{2}\left(v+v^{T}\right)=0
$$

since $u, v$ are vectors. Hence the norm of $q$ is constant.

\section{Acknowledgements}

The authors would like to thank Silvère Bonnabel for his help in the theoretical development and mathematical justification of the proposed filter.

\section{References}

\footnotetext{
${ }^{1}$ Martin, P. and Salaün, E., "An Invariant Observer for Earth-Velocity-Aided Attitude Heading Reference Systems," Proc. of the 17th IFAC World Congress, 2008, pp. 9857-9864.

${ }^{2}$ Martin, P. and Salaün, E., "A General Symmetry-Preserving Observer for Aided Attitude Heading Reference Systems," Proc. of the 47th IEEE Conf. on Decision and Control, 2008, pp. 2294-2301.

${ }^{3}$ Martin, P. and Salaün, E., "Design and Implementation of a Low-Cost Aided Attitude and Heading Reference System," Proc. of the 2008 AIAA Guidance, Navigation, and Control Conference, 2008, AIAA 2008-7169.

${ }^{4}$ Crassidis, J., "Sigma-point Kalman Filtering for Integrated GPS and Inertial Navigation," IEEE Trans. on Aerospace and Electronic Systems, Vol. 42, No. 2, 2006, pp. 750-756.

${ }^{5}$ van der Merwe, R., Wan, E., and Julier, S., "Sigma-point Kalman Filters for Nonlinear Estimation and Sensor-Fusion: Applications to Integrated Navigation," Proceedings of the AIAA Guidance, Navigation \& Control Conference, 2004, pp. 5120-5150.

${ }^{6}$ Hide, C., Moore, T., and Smith, M., “Adaptive Kalman filtering for low-cost INS/GPS,” The Journal of Navigation, Vol. 56, No. 01, 2003, pp. 143-152.

${ }^{7}$ Sasiadek, J., Wang, Q., and Zeremba, M., "Fuzzy adaptive Kalman filtering for INS/GPS data fusion," Proc. of the 2000 IEEE International Symposium on Intelligent Control, 2000, pp. 181-186.

${ }^{8}$ Song, Y. and Grizzle, J., "The Extended Kalman Filter as a Local Asymptotic Observer," Estimation and Control, Vol. 5, 1995, pp. 59-78.

${ }^{9}$ Shuster, M. and Oh, S., "Three-Axis Attitude Determination from Vector Observations," Journal of Guidance, Control and Dynamics, Vol. 4, No. 1, 1981, pp. 70-77.

${ }^{10}$ Lefferts, E., Markley, F., and Shuster, M., "Kalman Filtering for Spacecraft Attitude," Journal of Guidance, Control and Dynamics, Vol. 5, No. 5, 1982, pp. 417-429.

${ }^{11}$ Markley, F., "Attitude Error Representations for Kalman Filtering," Journal of Guidance, Control and Dynamics, Vol. 26, No. 2, 2003, pp. 311-317.

${ }^{12}$ Huang, Y., Chang, F., and Wang, L., “The Attitude Determination Algorithm Using Integrated GPS/INS Data," Proc. of the 16th IFAC World Congress, 2005 .

${ }^{13}$ Bijker, J. and Steyn, W., "Kalman Filter Configurations for a Low-Cost Loosely Integrated Inertial Navigation System on an Airship," Control Engineering Practice, Vol. 16, No. 12, 2008, pp. 1509-1518.

${ }^{14}$ Bonnabel, S., Martin, P., and Salaün, E., "Invariant Extended Kalman Filter: Theory and Application to a Velocity-Aided Attitude Estimation Problem," Proc. of the 48th IEEE Conf. on Decision and Control, 2009, pp. 1297-1304.

${ }^{15}$ Bonnabel, S., Martin, P., and Rouchon, P., "Symmetry-Preserving Observers," IEEE Trans. Automat. Control, Vol. 53, No. 11, 2008, pp. 2514-2526.

${ }^{16}$ Bonnabel, S., Martin, P., and Rouchon, P., “Non-Linear Symmetry-Preserving Observers on Lie Groups," IEEE Trans. Automat. Control, Vol. 54, No. 7, 2009, pp. 1709-1713.

${ }^{17}$ Bonnabel, S., "Left-Invariant Extended Kalman Filter and Attitude Estimation," Proc. of the IEEE Conf. on Decision and Control, 2007, pp. $1027-1032$.

${ }^{18}$ Stevens, B. and Lewis, F., Aircraft Control and Simulation, 2nd ed., 2003.
} 